\title{
Educational Management Systems in Secondary Education in Hordaland County, Norway Positional Paper
}

\author{
Dale Casey \\ Department of Education, Hordaland County, Norway \\ Dale.Ellen.Casey@hfk.no
}

\begin{abstract}
Educational management at the secondary school level in Norway is quite similar for all the counties in Norway. Not only are systems used in secondary education integrated, they are also integrated with many other systems used in government and education at various levels. This positional paper describes these systems and how they work together.
\end{abstract}

Keywords: Educational management system, school management system, learning management system, integration.

\section{Introduction}

The goal of this positional paper is to present the systems for educational management used in Hordaland County, Norway and to indicate how they are integrated, from a user point of view. Integration and interfacing of systems should be easy to achieve, but that is far from the situation we experience today. In Norway, we divide educational management systems (EMS) into two categories: school administrative systems (SAS); and learning management systems (LMS). Systems used for school administration contain functions for planning a school year, making schedules. A schedule would include a group of students, a subject and a teacher and in some instances a time and place. The EMS is also where official records are stored; these include semester grades, final grades, examination grades as well as attendance records and behavior grades. A learning management system is used in the classroom to implement the day to day business of education, like assignments and lessons and learning resources. This necessitates integration between the EMS and the LMS.

It is important to have a basic knowledge of the school system and the philosophy behind use of information and communication technology (ICT) in the schools, both for education and management. I start by giving a short description of the Norwegian educational system and Hordaland County. Next there will be a brief description of the major management systems in use followed by an explanation of how the systems interact. 


\section{Norway, Hordaland County and the Norwegian Educational System}

Norway is a small country with respect to population, but a very large country geographically with many remote areas. ICT has been instrumental in facilitating equal opportunity and access in all regions of the country, not least of all in education. When you only have a population of 5 million, coordination of data and systems is easy, or at least it should be. National initiatives are relatively easy to implement, ensuring pretty much the same structure which allows us to use the same system or systems with similar architecture, throughout the country.

Hordaland County is one of 19 entities that are separately responsible for education of secondary level students (15 to 18 year olds). Approximately $10 \%$ of Norway's population lives in Hordaland County, so we educate approximately $10 \%$ of the nation's youth. That means we have about 25,000 students, requiring 3,000 educators and 2,000 to 3,000 support personnel (maintenance, library, administration, etc.). The secondary schools in Hordaland County range in size from 100 students to over 1,000 students. Digitalization makes it possible to give equal education to all students.

There are 10 years of mandatory education, starting at the age of 6 . For the first 6 years of mandatory education, the students receive an evaluation twice per school year, but no grades are given. Grades are only given in the last 4 years of mandatory education (in junior high school). The grades are used to rank students when they apply for secondary education (high school) and the grades given in high school are used to rank applicants to higher education (university).

Further education is optional, but all youth have a right to free secondary education, vocational or college preparatory courses. Almost all Norwegian youth complete at least secondary education. The first two years of a vocational education take place in school after which there is a two year apprentice program administrated by the department of education in each county. At the end of the apprenticeship, a journeyman certificate is issued. (A journeyman is a person who has served an apprenticeship at a trade or handicraft and is certified to work at it, for example, an electrician.) There is also a special program where you obtain both college preparatory competency and a journeyman certificate. The two year apprenticeship is particularly useful for students planning on becoming engineers, ambulance personnel or nurses.

Education in ICT in primary and secondary education is called the $5^{\text {th }}$ skill. The 5 skills are: reading, writing, speaking, arithmetic and use of ICT. ICT is not taught as a separate subject. ICT competency is evaluated in each subject, assessing how well the students use the digital aids that are appropriate to the subject. We also have the National Digital Learning Arena whose sole function is to develop digital aids for the subjects taught in secondary school. Many other digital aids from other providers are also in use.

Since digital competency is a part of every subject, even more so in secondary school, students need a laptop available at all times. In Hordaland, we give each student a laptop. We use computers with Microsoft Windows operating system. Our supplier sets up and configures the computers in accordance with requirements we set. Students can freely download, as if the computer were their own. They pay a fee for 2 
or 3 years, after which they may keep the computer. The fee corresponds to the grant each student gets from the national government. Other counties have other solutions. Some allow their students to bring their own computer (Macintosh or personal computer (PC)) and, at the other extreme, other counties own and retain full control over the computer and only permit students to download pre-approved applications.

\section{Brief Description of Our Management Systems}

This section contains a short description of seventeen systems in use. Some of these are used in other areas, not just education. The * indicates a national database.

Residents' Data Base (Folkeregister)*. Folkeregister is the official national register containing (almost) every resident in Norway, with their address. This is "where you come into existence" in Norway. Your birth is registered and you are issued a person number. For non-nationals or immigrants, an application for a person number must be made, and a permanent one is only given if one has fulfilled certain criteria. All students wishing to pursue secondary education need a person number. However, there are many instances where a temporary person number is made up. Typical examples include exchange students or students in the process of applying for asylum.

Almost without exception, the person number is used to synchronize databases. In many instances the person number is used as in the identification data (ID) field of a database to uniquely identify a person. Examples of use are bank accounts, tax records, personnel databases and in this context, our SAS and our LMS.

Curriculum Data Base (GREP)*. The curriculum database is an official code book for education. It contains codes and curriculum information including the structure, study program codes, and course codes for all subjects taught in Norwegian secondary schools. In addition to the codes, the database contains 13,000 competency objectives for the courses taught as well as information on the examination form, number of teaching hours (credits), etc. These codes are imported to our SAS and LMS.

Application for Admission to Secondary Education (VIGO)*. VIGO is a nationally owned system for processing applications to secondary education. It is cordoned off to only permit application to secondary education in the county of residence, with a few exceptions for programs available for students in the whole country.

All application, processing of applications, assignment to a school and program and acceptance functions are electronic. Grades are imported from an SAS at the primary level. The grades are then weighted and assigned a point score. The sum of these points gives a score for overall achievement. This score is used to rank students.

Students must apply for school and a study program every year. They are allowed 3 choices and are guaranteed admission to one of these 3 choices. If they have completed a previous level of a particular study program, then they have to be admitted to one of the following programs from the same study program. It is not permitted to go on to a $2^{\text {nd }}$ or $3^{\text {rd }}$ year of a program until the previous level has been completed. 
Processing of applications is a 4-stage process. The first stage produces statistics about how many students have applied for what programs and where. This is used by the county department of education to make adjustments to the offerings at the various schools. If there are few applicants for a program at a particular school, then that program is dropped and those applicants have their application transferred to a school where the program will be held. If there are more applicants to a program than there are places, a new class may be created. The score for overall achievement is used to place students, those with the best grades, i.e. the best score, are placed first choice.

The second, third and fourth stages consist of: ranking students; assigning them to a school; informing them; and the applicants either accepting or declining. This is primarily done by system generated text messages (SMS). Students receive a message containing information on what they have been accepted for. They can either accept the placement or be put on a waiting list for another choice. Students who do not send an acceptance are no longer considered to be applicants. This process is repeated taking into account the new data supplied by the applicants - did they accept, change their priority or want to be on a waiting list. If they are on a waiting list, they can also accept admittance to a lower priority course and still be on a waiting list. The resulting data are exported to our SAS.

At the end of every school year, student progress is loaded into the VIGO system by our SAS. This is used for the next school year, for applying for higher education and to provide statistics for the national government.

Personnel and Payroll (PAGA). Every employee in Hordaland County starts their data trail in this system. It is also used for paying wages and transferring taxes to the treasury. There is a special code that indicates if an employee works at a school or the county department of education. This code is used to key export of personnel data to our SAS.

Student PC Administration (FrontEnd). Each student receives a laptop when they start school. The laptops are set up by the provider, given an ID number in the form of a barcode that is attached to the computer. The PCs are distributed directly from the supplier to our schools in accordance with the number of computers needed.

School Administrative System (Extens). Our SAS is called Extens and is a product developed in Norway. It is based on the Norwegian secondary school system. Any SAS should reflect the structure, functions, laws, rules and regulations of the educational system it is intended to manage. In Norway, education is controlled centrally, that is, the national government is responsible for all levels of education for private as well as public education. Thus the educational system for the whole country is homogeneous.

There are other systems in use in other counties in Norway, but there is little practical difference between them. For this reason, when it became time for a new SAS for secondary schools, the national government deemed it was time for one system in all the counties of Norway. This resulted in a document detailing the user demands for 
a new system and tenders have been obtained. Many complex aspects were uncovered and have been addressed.

The SAS has the following "modules": employee information and administration, for both teaching and non-teaching staff; student information and administration; school information and administration; semester administration; examination information; class administration; course codes (national standard) and requirements, both national and local, and administration; student assessment, attendance and behavior including reports; and administration of student PCs.

This system is the hub of all our other systems for use in the schools. This will be reflected in the section entitled "Integration" where the point of perspective considers how this system links and integrates to and from other systems.

System for Schedule Planning (UNTIS). Our SAS does not have a very useful module for schedule planning activities. This necessitates another system being integrated into the mix. Course codes, classes, teachers and students are joined together to produce necessary schedules. This system produces hypertext transfer protocol (http) files that can then be published on school web pages.

Access (AD - Active Directory). Each user is given a unique username and password in our directory (AD). This gives access to a network and an email account. Personnel receive this as a result of being registered as an employee. Students receive this as a result of being admitted to one of our schools.

Authentication (Feide)*. The username and password as well as the person number are sent to a central access function called Feide. Feide makes single sign on possible as a function for all your applications. All end user systems provided by a county or city must use this central sign on system. You can either sign on directly to Feide or you can sign on to one of the applications you have access to. We use Feide for access to our LMS.

Email System for Employees and Email System for Students. Each employee and each student has an email account through Hordaland County. These email accounts are created automatically, directly following creation of a user in our directory. Username and password for logon to an email account is the same as the logon to all other systems.

There are two separate email systems, one for students and one for employees. There are positive and negative sides to this separation. On the plus side, all employees are found in the same email address book, whether they are work at a school or at the central administration. On the negative side, students and only students are found in the student email address book. That makes it difficult for a teacher to send an email to a student and vice versa.

Learning Management (LMS - “Its learning"). We use a learning platform called "Its learning". "Its learning" is also the name of the company that provides the system 
and this company is based in Norway. It is the most popular system in use in Norway at the secondary level. The system is in use in countries outside Norway, like the Netherlands and the UK. There are versions for elementary school, secondary school and higher education.

The LMS is used to organize daily activity in the classroom, including courses with teachers and students. Since this information is already contained in our SAS, it is imported as is to the LMS. The teacher can then create teaching plans, activities, lessons, tests and keep track of student progress, effort, behavior and attendance. It seeks to offer everything a teacher needs, every day, for implementation of the goals for a course.

In addition to the functions built into the system, there is an extensive applications library including Britannica Online, Atekst (a digital newspaper archive), NRK skole (television programs from the Norwegian broadcasting agency), NDLA (see the next sub-section), etc. Currently there are 28 applications; some of these require a license fee. "Its learning" has formal agreements with Microsoft and Google so these products can also be used from within the system.

National Digital Learning Arena (NDLA)*. The NDLA was established when the Norwegian counties (all except one) decided to pool a considerable amount of their funds for teaching resources to create digital learning aids for use in secondary education. These resources are open and free to use. They currently encompass general studies courses (subjects found in all study programs) like Norwegian, English, mathematics, social studies, physical education and general science. There are several subject areas for college and university preparatory courses and 9 (of 13 available) subject areas for vocational trades. These resources are available for use from inside our LMS as well.

Documentation of Student Progress (SkoleArena). SkoleArena is a system for documentation of student progress. SkoleArena contains the official record of all work, evaluation, attendance, behavior and examination results. Attendance, behavior and examination results are entered directly into SkoleArena as is the semester grade. Grades for classroom activity may also be entered manually, but our policy is that these grades be registered in our LMS and imported.

Students can log on to this system (or click on a link from our LMS) to see their progress in each of their courses or their attendance and behavior records. Teachers have access to information for the students they have in their class and in the subjects they teach.

System for Examination Administration and for Implementation of Digital Examinations (PAS/PGS)*. Examinations are submitted for evaluation and grading anonymously so this system (PAS) is used to assign a candidate number to each student for each examination they will sit. We have many digital examinations that are standard for the whole country, like Norwegian, English and mathematics. These 
examinations are found on PGS. When a student submits a digital examination, it is also evaluated in its digital form. There are 2 examiners for each examination. Each examiner opens the examination and makes their evaluation and sets a grade. If the examiners disagree on the grade, they discuss it and agree on the final grade to be given. Digital examinations save time and money. Sending examination papers through the mail to and from remote areas of the country takes time and is costly, not to mention the risk of losing them along the way. Examination grades are entered in SkoleArena and exported to our SAS.

National Certificate Database (Graduation Certificate/Journeyman Certificate)*. The purpose of the National Certificate Database is to maintain a record of all certificates and transcripts of grades issued from secondary schools. The database goes back to 2000. Our SAS produces a paper copy for the recipient. At the same time, it sends an electronic copy to this database. Journeyman certificates will become a part of this system in 2013.

Application for Admission to Higher Education (Samordnet opptak)*. The system for application to higher education has the same purpose as the system for admission to secondary school. Higher education is university or college education. Those wishing to pursue higher education register their application electronically. All institutions of higher education are included. The applicant registers which school they wish to attend and which course of study they wish to pursue. Acceptance is based on points for performance in secondary school. Certain courses of study require specific subjects and a minimum grade in order for the application to be considered. For example, language studies require the student to have completed the language they wish to study in secondary school; medical students need chemistry and biology as well as mathematics.

Learning Portal (Skoleportalen). Skoleportalen is a website owned and operated by Hordaland County. The tool used for creating templates and publication of pages is Episerver. We provide the schools with a set of master templates that they personalize to fit their own school's needs. Editors are given access to edit their own school's pages through the access system (AD) in the county.

Our learning portal was established in 2000 and has had the same function and appearance since. The learning portal is intended to be an electronic door into schools and activities in the school. These activities include LMS, mail and a pull-down menu with a list of school websites. In addition, there is a rather extensive pointer collection, broken down into the various study programs. It is possible to search the pointer collection as there are meta-tags as well as the study program breakdown.

\section{Integration - Not Really, It's Just Import}

This section will explain how our systems are integrated or, rather, interfaced. We have no true integrations; we send copies of data from one system to others. Updates 


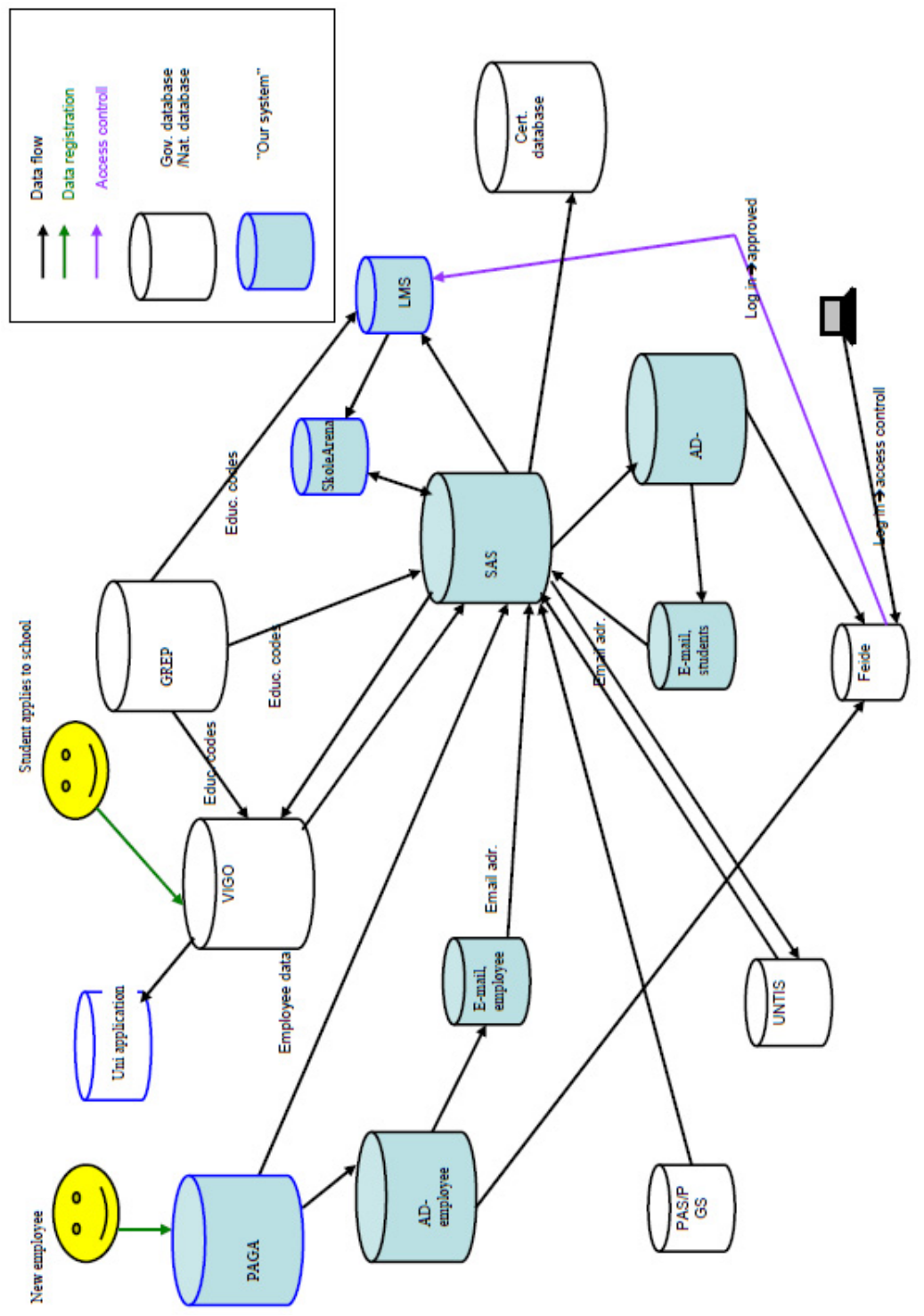

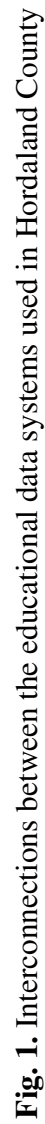


in the receiving system(s) do not result in updates in the original. When data in the original are changed, they are re-imported. This results in the overwriting of any changes that have been made at the receiving end.

In the following sections, the systems are explained as pairs, with the exception of the first few sub-sections. Since our key system is our SAS, it will be used as a core from which the other systems are described in terms of their connections. At the end, how all the systems are interfaced will be illustrated (see Figure 1).

GREP and Other Systems. One system that is used with many other systems is GREP. GREP is the national code database and these codes are imported to many systems. Many of the systems the codes are imported to allow additions and changes at user discretion. Unfortunately, changes in one system are nontransferable to other systems, causing inconsistency between systems.

The following systems use these codes (note use is independent between the systems they are used in and data flows only one way, from GREP): VIGO (application for admission to secondary education, uses original codes only); Extens (SAS); "Its learning" (LMS); NDLA (digital aids); SkoleArena (documentation of student progress); National Certificate Database (uses original codes only); PAS/PGS (system for examinations, uses original codes only); Samordnet opptak (application for admission to higher education, uses original codes only).

SAS and "Folkeregister". The first pair of systems is our SAS and Folkeregister (resident database). There is no direct connection between these systems, but the person number used in our SAS has to be identical to the one used in the Folkeregister. However, it is possible to register people manually in the SAS when they do not have a person number. In these cases, the number is made up. If a permanent number is issued, the number created in the SAS has to be changed.

VIGO and SAS. Data from the VIGO system (for application to high school) is imported to the SAS, including assignment of school and program of study. At the end of a school year, student assessments are exported from the SAS to VIGO. This is necessary for the next year of application, whether it be for further secondary education or higher education. This is not an integration, but a transfer of data first from VIGO to the SAS and then from the SAS to VIGO.

PAGA and SAS. Employees are registered in our personnel and payroll system PAGA. If they are to work in a school or at the department of education, they are imported to our SAS. The personal data imported to the SAS is complete except for the details of their daily work tasks. The school or department name is also imported to the SAS.

FrontEnd and SAS. Our supplier of student PCs registers each PC in FrontEnd (the PC administration system). The supplier also attaches an ID sticker with a barcode to each PC prior to delivery and distribution. When a student comes to pick up the PC, the consultant accesses the student in the SAS and scans the barcode. All computer 
information is then imported to the SAS and it is coupled with the student that will be using it. It also facilitates collection of the fee for using the computer by producing a bill and the student must also sign a contract (or the parents must sign it if the student is under the age of eighteen).

UNTIS and SAS. The next system pair is UNTIS (schedule planning) and SAS. Course information is imported to UNTIS where schedule planning is completed. The plans may then be imported to a school website. Other useful functions included are calculation of workload in order to assure that employees have the same amount of work that they are being paid for and that students have the correct courses and amount of coursework.

SAS and AD. When a student user is created in the SAS, a command goes to the AD (access control) asking it to create a user. The user is created and given a username and password for email, network and various other systems. Employee users are created in connection with registration in the personnel system. When a student has finished high school, a command goes from the SAS to AD with this information. When an employee terminates their position, the personnel system sends this information to $\mathrm{AD}$. The user accounts are put into an inactive status where they remain for a year and a half before being deleted. The username cannot be used again until the inactive user has been deleted.

SAS and Email Systems. Both employee and student email addresses are created by the AD. This happens as soon as a username has been created in the AD. These are then imported to the SAS. The email address could be edited by the user via our SkoleArena, but we have turned off this function. It was necessary to deactivate this function due to incompatibility of the set of symbols and letters allowed in SkoleArena and the SAS. Certain symbols would cause the LMS import to fail and it would fail until the email address was corrected.

SAS and LMS. The next pair of systems is one of the most important. It is between the SAS and the LMS. At the beginning of a school year, all students should have an inactive status in the SAS. This means they are stored away in the trashcan of the LMS. Students that will continue to study are moved out of the LMS trashcan at the start of the next school year. The students that have finished their schooling are left in the trashcan until the period of quarantine is over. This is currently 18 months, after which they are deleted.

All courses from the previous school are deleted in the SAS. This causes them to lose their connection to the structure in the LMS and they are then transferred to the archive where they stay until a teacher retrieves them or deletes them. Furthermore, the hierarchy or structure from the previous year is deleted in the LMS as it no longer reflects the current structure of courses and classes. Some counties keep the structure of more than one school year, but we do not. All deletion from the trashcan in the LMS must be done manually.

At the start of a new school year, the schools make a complete course plan in the SAS. The course plan consists of subjects to be taught, who is taking them, in which 
group and who will be teaching them. Classes are also created with a "contact teacher" teacher and all the students. This structure is then imported to the LMS and a new school year is created. Each course and class in the SAS causes an LMS course or class to be created. Changes in students or teachers that are made in the SAS are automatically updated in the course or class groups.

SAS, SkoleArena and LMS. SkoleArena is an interface between the LMS and the SAS. It shows some of the data that is in the SAS. There is additional data stored only in SkoleArena. Some of the data in SkoleArena are concatenated and stored in the SAS. This data includes attendance, behavior, semester grades and examination grades. The fields that are available for direct edit are actually fields in the SAS. Examples of this are email address and telephone number. Most schools have chosen to lock these fields for their students to avoid them registering garbage.

SkoleArena also receives data from the LMS. Our LMS enables all everyday learning activities to be done digitally. This includes tests and course work. The teacher evaluates and comments on the work and sets a grade or approved/not approved status on the work. These evaluations are then exported from the LMS to SkoleArena.

SAS and PAS/PGS. The next pair of systems is PAS/PGS (examination system). Examination data is imported to the SAS. This includes examination codes, student identification (person number), examiner identification (person number) and the date of the examination.

SAS and Certificate Database. The SAS and the certificate database are also connected. Examination grades as well as behavior and attendance are printed out for the student at the end of each semester and school year. This certificate is also sent to the central database from the SAS.

Samordnet Opptak, VIGO and Certificate Database. At the end of a school year, all grades are transferred from the SAS to VIGO. They are then sent to the national government (bureau of statistics). If the student is applying for higher education, the results also go to the system used for application for admission to higher education (Samordnet opptak). Samordnet opptak also uses data from the certificate database.

AD and Other Systems. Our AD (user directory) has many functions. When our AD creates a user, an email account is automatically created. Editors of school websites are given access to their school's website in our AD. The users are then imported to the website and may sign in with their same username and password. The AD is also connected to "Feide", enabling single sign on to 19 applications.

\section{Conclusion}

No computer systems in Norway can be called "large systems"; there are not enough data records. However, the amount of data is no longer relevant, since storage and 
retrieval of data is the simplest part of a system. The largest system is of course the "Folkeregister" with 5 million people registered. The systems are none-the-less very complex and there are many connections that have to communicate with one another. This is where the issue lies; data only flows one-way. The systems end up with corrupt data. Data that was identical to begin with gets changed in one system and the changes do not go back up the line to the source. Sometimes this causes a break in synchronization so transference to other systems down the line becomes impossible.

Glitches in source data create problems in the integrated systems that have to be corrected manually, but you have to find them first. In one instance, a huge amount of "garbage" was sent to our SAS from the national database due to an error they had. The changes in our SAS caused our LMS to try and create 8,000 new courses. Fortunately we had a safeguard on and stopped the import. Had it gone through, all of these courses would have been garbage, but they would have required manual deletion and a quarantine period of 18 months before they could be permanently deleted.

Data imported from the same source to different systems is not always identical in the various systems. The SAS and the LMS import data from the national subject database, independent of each other. Each of these systems allows for supplementation with local goals and evaluation criteria. Since there is no synchronization of this information, this is at best double work, and in the worst case they are not the same. When grades/evaluations are imported from the LMS to SkoleArena, goals and evaluation criteria do not pass over. The teacher then has to go into SkoleArena and connect up the same goals and evaluation criteria that they had available in the LMS.

There are many examples of "where it can go wrong" and it does almost every day. These kinds of errors are time consuming to find and they result in low quality data. For the most part it works astonishingly well. In a perfect world, we would have one central pool of data with applications drawing from it. Unfortunately, our system providers do not have the same philosophy.

Acknowledgements. The author would like to thank: colleagues at Hordaland County for valuable feed back in connection with this paper and the presentation made at the ITEM 2012 conference in Bremen; and Don Passey, Senior Research Fellow, Department of Educational Research, Lancaster University, Lancaster, LA1 4YD for encouragement and help in preparing this paper for publication. 